\title{
AUCTIONING MANY DIVISIBLE GOODS
}

Lawrence M. Ausubel

University of Maryland
Peter Cramton

University of Maryland

\begin{abstract}
We study the theory and practical implementation of auctioning many divisible goods. With multiple related goods, price discovery is important not only to reduce the winner's curse, but more importantly, to simplify the bidder's decision problem and to facilitate the revelation of preferences in the bids. Simultaneous clock auctions are especially desirable formats for auctioning many divisible goods. We examine the properties of these auctions and discuss important practical considerations in applying them. (JEL: D44)
\end{abstract}

\section{Introduction}

Auctions of divisible goods are commonplace, especially in markets for financial securities, energy products, and environmental permits. In such auctions, the bids specify quantities of the divisible goods: The shares of stock, the megawatt-hours of electricity, or the tons of emissions. Often, related goods are-or could be-auctioned at the same time. For example, government securities are typically offered in a variety of durations. In electricity markets, products with several durations or locations may be auctioned together. In environmental auctions, emissions for each of several different pollutants or time periods may be sold at the same time. This article explores how auctions for many divisible goods should be conducted. Of course the answer depends on the objective of the seller and the bidding environment. ${ }^{1}$ Here we focus on a few of the important issues of auction design in a setting where the seller cares about some combination of efficiency (assigning the goods to the bidders who value

Acknowledgments: We are grateful for the support of National Science Foundation Grants SES-01-12906 and IIS-02-05489. Authors' web sites: 〈www.ausubel.com〉 and 〈www.cramton. umd.edu $\rangle$. Further materials on the theory and practical implementation of auctions are available on the web sites of Market Design Inc. (www.market-design.com) and Efficient Auctions LLC $\langle$ www.efficientauctions.com $\rangle$, as well as in the recent book by Milgrom (2004).

E-mail addresses: Ausubel: ausubel@econ.umd.edu; Cramton: cramton@umd.edu.

1. As is common in auction theory, we will study dynamic auctions to sell, in which the auctioneer is a seller, the bidders are buyers, and the price typically ascends. Analogous results apply in auctions to buy (i.e., procurement), in which the auctioneer is a buyer, the bidders are sellers, and the price typically descends. 
them the most) and revenue maximization. Our purpose is to motivate a sensible design in a realistic environment, rather than to prove the optimality of a particular design, which would require stronger assumptions than we care to make.

One of the initial design decisions is whether to conduct a static (sealed-bid) or dynamic (ascending-bid) auction. A frequent motivation for the use of dynamic auctions is reducing common-value uncertainty, thereby enabling bidders to bid more aggressively with less fear of the "winner's curse." However, in the context of selling many goods, the price discovery of a dynamic auction plays another, often more important, role. By seeing tentative price information, bidders are better able to make decisions about the quantity of each good to purchase. This is useful because the goods being sold are related. Some may be substitutes; others may be complements. Bidding in the absence of price information makes the problem much more difficult for bidders. Furthermore, practical realities such as budget constraints can make bidding in a sealed-bid auction exceedingly difficult unless the auctioneer allows the bidders to express these constraints in their sealed bids; whereas, in a dynamic auction, the bidder can see tentative prices and assignments, allowing the bidder to make decisions that are consistent with the bidder's constraints.

The case for dynamic auctions is further strengthened when we recognize that it is costly for bidders to determine their preferences. A dynamic auction, by providing tentative price information, helps focus the bidder's decision problem. Rather than consider all possibilities from the outset, the bidder can instead focus on cases that are important given the tentative price and assignment information. Although this point is already valid in auctions for a single good (Compte and Jehiel 2000), it becomes more critical in the context of many goods, where the bidder's decision problem is much more complicated. Rather than simply decide whether to buy, the bidder must decide which goods to buy and how many of each. The number of possibilities grows exponentially with the number of goods. Determining values and then bids for each of these possibilities is difficult at best; whereas, in the presence of good price information the decision problem becomes relatively straightforward.

Given the increased importance of price discovery when auctioning many divisible goods, we focus on dynamic auctions. The question then becomes: How can the auction designer best promote effective price discovery? Simultaneous clock auctions are both effective and simple. In a simultaneous clock auction, there is a price "clock" for each divisible good indicating its tentative price per unit quantity. Bidders express the quantities desired at the current prices. The price is incremented for goods with excess demand, and bidders again express their desired quantities at the new prices. This process repeats until demand is made equal to supply. The tentative prices and assignments then become final. 
We begin with two stylized models that abstract away from many of the complicating practical details. In each, we assume that there is a continuum of bidders, so that market power considerations can be ignored. We also assume that the bidding occurs in continuous time, avoiding issues arising from discreteness. We show that the equilibrium outcome of the auction coincides with the competitive equilibrium of the model and attains full efficiency. In this sense, the simultaneous clock auction brings to life the "Walrasian auctioneer" often used to motivate the competitive equilibrium.

We then turn to practical considerations that need to be addressed in any real-world situation.

Discrete rounds, rather than bidding in continuous time, means that issues of bid increments, ties, and rationing are important. We argue that this complication is best handled by utilizing "intraround bids," allowing bidders in each round to express their demand curves along a line segment between the starting and ending price for the round. Allowing a rich expression of preferences within a round makes bid increments, ties, and rationing less important. Since preferences for intermediate prices can be expressed, the efficiency loss associated with the discrete increment is less, so the auctioneer can choose a larger bid increment, resulting in a faster and less costly auction process.

Natural linkages among goods often exist in practice. For example, in the case of an auction of electricity capacity, the goods may differ by the duration of the contract (e.g., three months, one year, or multiple years). Such products are natural substitutes: A two-year contract is simply a sequence of two one-year contracts. Hence, the relative prices of such products are closely related. The auction can exploit this linkage by enhancing substitution possibilities across these products.

Market power is a final practical consideration. Although some auction settings approximate the ideal of perfect competition, most do not. The auction design needs to address limited competition. Three useful instruments are information policy, reserve pricing and efficient pricing. By controlling the information that bidders receive, the auctioneer can enhance price discovery while limiting the scope for collusion. Reserve pricing serves two roles, providing price discipline in the absence of competition and discouraging collusion by limiting the maximum gain from successful collusion. Finally, since uniform pricing inevitably leads to demand reduction (Ausubel and Cramton 2002), the resulting inefficiency can be avoided by instead using the efficient pricing rule of the Ausubel (1997) auction.

We now address each of these issues in detail. Section 2 develops theoretical results in a continuous-time model with perfect competition. The article then turns to considerations of practical implementation, treating discrete rounds (Section 3), natural linkages among goods (Section 4) and limited competition (Section 5). 


\section{Auctioning Many Goods in the Absence of Market Power}

In this section, we specify two economic models in which the simultaneous clock auction can be shown to yield desirable outcomes. The final prices of the auctions correspond to the competitive equilibria of the models, and the allocations are therefore efficient. The models abstract from reality in their use of a continuum of bidders (justifying price-taking behavior) and continuous time (allowing the price adjustment processes to converge perfectly).

A seller wishes to allocate units of each of $K$ different goods among a continuum of bidders. The bidders are subscripted by $i(i \in[0,1])$. Each bidder $i$ 's consumption set, $X_{i}$, is assumed to be a compact, convex subset of $\mathbb{R}_{+}^{K}$, and bidder $i$ 's consumption bundle is $x_{i}=\left(x_{i}^{1}, \ldots, x_{i}^{K}\right) \in X_{i}$. The supply available is $S=\left(S^{1}, \ldots, S^{K}\right) \in \mathbb{R}_{++}^{K}$.

In Model 1, we assume: ${ }^{2}$

(A1) Pure private values: Bidder $i$ 's value, $U_{i}\left(x_{i}\right)$, for consumption vector $x_{i}$ does not change when bidder $i$ learns other bidders' information.

(A2) Quasilinearity: Bidder $i$ 's utility from receiving the consumption vector $x_{i}$ in return for the payment $y_{i}$ is given by $U_{i}\left(x_{i}\right)-y_{i}$.

(A3) Monotonicity: The function $U_{i}: X_{i} \rightarrow \mathbb{R}$ is increasing.

(A4) Strict concavity: The function $U_{i}: X_{i} \rightarrow \mathbb{R}$ is strictly concave.

The price vector is denoted by $p=\left(p^{1}, \ldots, p^{K}\right) \in \mathbb{R}_{+}^{K}$. If each bidder $i$ demands $x_{i}(p)$ at price vector $p$, the aggregate demand is given by $\int_{0}^{1} x_{i}(p) d i$. The excess demand, $z(p)$, equals the difference between the aggregate demand and the supply: $z(p)=-S+\int_{0}^{1} x_{i}(p) d i$. In Walrasian tâtonnement (Walras 1874, as formalized by Samuelson 1941), the price vector adjusts in continuous time according to excess demand: $\dot{p}(t)=f(z(p(t)))$, where $f(\cdot)$ is a continuous, sign-preserving transformation. The price vector $p$ and the associated demands form a competitive equilibrium if the excess demand equals zero for every good.

The following auction procedure is followed:

- the auctioneer announces a price vector $p$;

- bidders report demands $x_{i}(p)$;

- the auctioneer adjusts the price vector according to Walrasian tâtonnement; and

- the process repeats until the price vector converges.

We have the following result for Model 1 :

2. In addition, we assume away any arcane difficulties that would unnecessarily complicate the analysis. In particular, we assume that the bidders' demands, $x_{i}(p)$, are measurable with respect to $i$. This is easily justified if the continuum of bidders consists of a finite collection of subintervals of bidders and, within each subinterval, the bidders are identical. It is also assumed that the consumption sets of the bidders and the available supply $S$ are such that there exists a feasible allocation of $S$ among the bidders. 
THEOREM 1. In Model 1, sincere bidding by the bidders is an equilibrium of the auction game and, starting from any price vector, the outcome converges to the competitive equilibrium.

Proof. A standard global stability argument (see, for example, Varian 1981). Let $V_{i}(\cdot)$ denote bidder $i$ 's net indirect utility function: $V_{i}(p)=\max _{x_{i} \in X_{i}}\left\{U_{i}\left(x_{i}\right)-\right.$ $\left.p \cdot x_{i}\right\}$. Define the following Lyapunov function: $V(p)=p \cdot s+\int_{0}^{1} V_{i}(p) d i$. Then: $\dot{V}(t)=\left(S-\int_{0}^{1} x_{i}(p(t)) d i\right) \cdot \dot{p}(t) \leq 0$, and $V(\cdot)$ is minimized at the competitive equilibrium.

Furthermore, with slightly stronger assumptions, the price adjustment is everywhere increasing:

COROLlaRY 1. In Model 1, if goods are substitutes in the aggregate, then sincere bidding by the bidders is an equilibrium of the auction game and, starting from any sufficiently small price vector, the price ascends monotonically to the unique competitive equilibrium.

It is not necessary for the goods to be substitutes, in order for a simultaneous clock auction to yield the desired outcome. Indeed, in the second model developed, the goods can be demanded in fixed coefficients-a most extreme form of complements. This result may appear counterintuitive in view of the literature on the Walrasian tâtonnement process. However, it should be emphasized that this traditional price adjustment literature proceeds with one hand tied behind its back; the adjustment process is restricted to depend only on the excess demand function, that is, to take a form that is economically plausible in the absence of any auctioneer. By contrast, for current purposes, there is assumed to be a real auctioneer, who we allow to exploit any information generated by the auction process (e.g., the gradient of the excess demand function) to facilitate convergence.

In Model 2, we will again posit a continuum of bidders and we will require that a unique competitive equilibrium exists. This time, we make assumptions on the aggregate demand function $x(\cdot)$ (as a function of $p$ ), instead of directly on the primitives of the model. We assume (A1) and the following:

(A2') Continuous differentiability: The aggregate demand function, $x(\cdot)$, is continuously differentiable at all $p \in \mathbb{R}^{K}$.

(A3') For every subset, $J$, of the set of all goods, and for every $p \in \mathbb{R}_{+}^{K}$, there exists $\alpha(p) \in \mathbb{R}^{K}$ such that, if $\dot{p}=\alpha(p)$ :

$$
\dot{x}^{j}(p)=0 \text {, for all } j \in J \text {, but } \dot{x}^{k}(p) \leq-1 \text {, for all } k \in K \backslash J .
$$

Assumption (A3') states that there is always a direction in which one can adjust price such that specified components of aggregate demand remain constant 
while the remaining components decrease at a rate bounded away from zero. An example of a model that satisfies these conditions is a model where $K=2$ and each of the bidders has Leontief (i.e., fixed coefficient) preferences, and where the fixed coefficients for valuing the two goods are in different ratios for different bidders. In such a model, it will generally be possible to raise the price of one good while lowering the price of the other good in such a way that the aggregate demand for the first good is constant but the aggregate demand for the second good decreases.

An auction procedure similar to that used for Model 1 may be followed, except that a different price adjustment process is used. We have the following result for Model 2:

THEOREM 2. In Model 2, with an appropriate price adjustment process, sincere bidding by the bidders is an equilibrium of the auction game and, starting from any price vector with positive excess demands for every good, the outcome converges to the competitive equilibrium.

Proof. Begin by increasing the price of every good until some good clears. Inductively, let $J$ denote the set of goods that have already cleared. Then adjust the prices so that the aggregate demands of all goods in $J$ remain constant, while the aggregate demands of all goods in $K \backslash J$ decrease. Assumption (A3') guarantees that it is always possible to adjust prices in this way, reducing the excess demand of all goods that have not yet cleared while maintaining market clearance for all goods that have already cleared. In finite time, some good $k \in$ $K \backslash J$ will clear. Set $J$ is then redefined to include good $k$, and the process is repeated. Clearly, all goods will clear in finite time.

REMARK. The adjustment process used in the proof of Theorem 2 is a simple version of the "sign process" of Laan and Talman (1987). More complicated complements preferences can also be accommodated within their adjustment process.

\section{Accommodating Discrete Rounds with Intraround Bidding}

Although in theory one can imagine implementing an ascending auction in continuous time, this is hardly ever done in practice. Clock auctions inevitably use discrete rounds for two important reasons. First, communication is rarely so reliable that bidders would be willing to be exposed to a continuous clock. A bidder would find it unsatisfactory if the price clock swept past the bidder's willingness to pay because of a brief communication glitch. Discrete rounds are robust to communication problems. Discrete rounds have a bidding window of 
significant duration, rarely less than ten minutes and sometimes more than one hour. This window gives bidders time to correct any communication problems, to resort to back-up systems, or to contact the auctioneer and have the round extended. Second, a discrete round auction improves price discovery by giving the bidders an opportunity to reflect between rounds. Bidders need time to incorporate information from prior rounds into a revised bidding strategy. This updating is precisely the source of price discovery and its associated benefits.

It is only in sequential descending clock auctions (Dutch auctions) that a nearly continuous bidding process is used. This is seen in Dutch flower auctions, many fish auctions, and U.S. tobacco auctions since 2003. All of these auctions are on-site (avoiding communication difficulties) and descending clock (eliminating any role for price discovery within the auction, since there are no events to condition on).

An important issue in discrete-round auctions is the size of the bid increments. Larger bid increments enable the auction to conclude in fewer rounds, but they potentially introduce inefficiency from the use of a coarse price grid. Large increments also introduce incentives for gaming as a result of the expanded importance of ties and rationing rules. But using small increments especially in an auction with many clocks can greatly increase the number of rounds and, hence, the time required to complete the auction. Bidders generally prefer a shorter auction. A short auction reduces participation costs. A short auction also reduces exposure to price movements during the auction. This is especially relevant in securities and energy auctions for which there are active secondary markets in close substitutes, and for which underlying price movements could easily exceed the price increments.

Fortunately it is possible to capture nearly all of the benefits of a continuous auction and still conduct the auction in a limited number of rounds, using the technique of intraround bids. ${ }^{3}$ With intraround bids, bidders express their demands in each auction round at all price vectors along the line segment from the start-of-round price to the end-of-round price. In a traditional clock auction, price may increase from say $\$ 90$ to $\$ 100$ in a round, but the bidder is only able to express the quantity it desires at $\$ 90$ and at $\$ 100$. With intraround bids, the bidder expresses its desired quantity at all prices between $\$ 90$ and $\$ 100$. This avoids the inefficiency associated with a coarser price grid. It also avoids the gaming behavior that arises from the increased importance of ties and rationing with coarser prices. The only thing that is lost is the within-round price discovery. However, within-round price discovery is much less important than the price discovery that occurs between rounds.

3. Intraround bidding, activity rules, indifference tables, and other aspects of the practical implementation of clock auctions are described in greater detail in Ausubel, Cramton, and Jones (2002). 
The experience from a number of high-stakes clock auctions indicates that intraround bidding lets the auctioneer conduct auctions with ten or more products in about ten rounds, with little or no loss from the discreteness of rounds. These auctions can be completed in a single day. By way of contrast, early spectrum auctions and some electricity auctions without intraround bids took weeks or even months to conclude. In a few extreme instances, the longer duration was warranted due to the enormous uncertainty and extremely high stakes, but generally speaking, intraround bids would have reduced the bidding costs without any meaningful loss in price discovery.

\section{Exploiting the Natural Linkages among Goods}

The motivation for auctioning many products together is that the products are related. The bidders may value some of the goods as substitutes and others as complements. At the same time, there may be substitution possibilities as to what is supplied in the auction. Given the natural linkages among goods, a second important issue in the implementation of a simultaneous clock auction is the amount of flexibility given to bidders in switching across products and to the seller in the determination of supply.

\subsection{Bidder Flexibility}

To promote price discovery, activity rules are generally imposed in clock auctions. The simplest clock auction is for a single homogeneous good. There, the activity rule takes the simple form of a monotonicity constraint: each bidder's quantity demanded is not permitted to increase as the price increases, consistent with downward sloping demand curves. Without the monotonicity constraint, a bidder might bid as a "snake in the grass"- grossly understating demands at low prices and then jumping in with large demands near the end of the auction. Widespread use of a snake-in-the-grass strategy would undercut the very purpose of utilizing a dynamic auction. ${ }^{4} \mathrm{~A}$ monotonicity constraint prevents this form of strategic behavior, thus encouraging better price discovery and facilitating rapid convergence to equilibrium.

In situations with multiple goods that have relatively independent demands,

\footnotetext{
4. One motivation for a bidder to use a "snake-in-the-grass" strategy is to avoid conveying information to rivals in an environment where bidders exhibit interdependent values. If each bidder's estimate of value is based in part on rivals' information, one bidder demanding large quantities might induce her rivals to raise their value estimates and bid more aggressively. A second motivation for a bidder to use a snake-in-the-grass strategy arises from budget constraints. The bidder holds back on bidding for the good she wants most dearly, instead bidding for the goods her rivals want, in the hopes of exhausting the competitors' limited budgets. The bidder then shifts to bidding on her true interests late in the auction, now facing weakened competition for these goods.
} 
a monotonicity constraint may be applied independently to each good. However, in situations where the interdependencies across goods are substantial, applying monotonicity constraints independently to each good may be overly restrictive. For example, if two products are close substitutes, it is natural for the bidder to demand the product with the more attractive price. Thus, the bidder may want to decrease the quantity she bids on the product with a faster rising price, and increase her quantity on the product with a slower rising price. Such bids would be excluded by the simplest application of monotonicity constraints.

In some applications, identifying and exploiting the natural linkages among goods may resolve these issues. Goods are organized into product groups. Substitute goods are assigned to the same group; while complementary goods are assigned to different groups. The activity rule is crafted to permit bidders to freely substitute among goods contained in the same product group. However, monotonicity is applied independently across groups, so that no substitution is permitted between products in different groups.

The quarterly Electricité de France (EDF) Generation Capacity Auctions, the first practical implementation of simultaneous clock auctions, has successfully taken this approach. Broadly speaking, two types of goods are offered: baseload capacity contracts; and peakload capacity contracts. These goods would be expected to be complements, since a new entrant in the French electricity market can best meet the needs of customers with a particular combination of baseload and peakload capacity. However, baseload and peakload capacity are each offered in multiple durations-three-month, six-month, one-year, two-year, and three-year contracts, with the same starting date-and the various durations of the same type of contract are close substitutes. Consequently, the goods are organized into two product groups, each containing five products. Since the goods within a group are denominated in comparable units (MW of power), the activity rule applied to all products within a group is simply a monotonicity constraint on the sum of the respective demands. ${ }^{5}$

\subsection{Supply Flexibility}

In many applications, the evident substitutability of goods makes it desirable to give flexibility to the seller, as well as to bidders. For example, government securities may be offered in a variety of durations, but the only real constraint on the seller is that the total quantity sold must equal the government's current debt requirements. Or energy products may be offered with a variety of delivery

5. As many as five product groups have been offered in some of the EDF auctions. An additional Power Purchase Agreement product has sometimes been offered, in addition to the basic baseload and peakload product. Moreover, in some of the auctions, contracts with different starting dates have been offered as separate product groups. For further information, see EDF's web site $\langle$ www.edf.fr $\rangle$ and the sources listed in the introductory footnotes. 
locations, but the principal requirement is again on the total quantity sold. The objectives of efficiency and revenue maximization are both served by allowing "the market to decide" the supply of each of the substitute products to be sold.

Again, the EDF auctions have successfully taken this approach. As described in Section 4.1, both baseload and peak capacity are offered in many durations. EDF recognized that different bidders would prefer different durations, but EDF did not have a reliable method for predicting the demands for the various durations other than through the auction itself. By way of contrast, EDF had excellent information about its own willingness to substitute quantities among durations, as a function of price.

Observe that, if both the supplies and the relative prices of the various durations were allowed to be determined endogenously, then the entire system would be underdetermined. Since the supplies were intended to be market driven and since the seller's trade-offs on price were well understood, the decision was made that the prices of the various products within a group would be linked together and would increase in lockstep. (However, the prices associated with different product groups move independently of one another.) Before the start of the auction, the seller determines an indifference table expressing the price differentials (i.e., a yield curve) amongst the various products within a group that would make the seller indifferent between selling one product or another. With two product groups containing five products each, there are effectively just two clocks (baseload and peak), although ten prices, with the prices for each product group determined by the clock and the indifference table. The clearing condition is that the aggregate demand for each product group is no greater than the total supply offered. The bidders then determine endogenously the division of sales across the various durations, contributing both to efficiency and to revenue maximization.

\section{Addressing Limited Competition}

In most auctions, competition is limited. Either the number of bidders is small or some bidders are significant in size relative to the auction volume. In these auctions, the auction designer needs to address the potential exercise of market power in the auction design. Three of the most important instruments available to the auction designer are: The information policy, the use of reserves, and the pricing rule.

\subsection{Information Policy}

In a competitive auction, price is used to resolve the assignment problem. Those bidders willing to pay more, get more. However, when there is a lack of 
competition, a major concern is that bidders will agree on a division of the goods by means other than the auction price.

Most spectrum auctions have used a fully transparent simultaneous ascending auction, in which the complete history of bids (including the identities of the bidders making the bids) has been reported after every round. This has enabled bidders to adopt collusive strategies in spectrum auctions where competition was especially weak (Cramton and Schwartz 2002).

In clock auctions, a useful information policy for mitigating collusive possibilities is to report only the aggregate demands for the goods after each round. In many situations, the aggregate demands contain most of the information needed for price discovery. If, instead, the auctioneer revealed the individual demands of the bidders, this detailed information could be used to facilitate a coordinated reduction of demands at low prices. For example, the bidders might cooperatively reciprocate the quantity reductions of competitors, and punish those who do not reciprocate by shifting quantity toward products most desired by the nonreciprocating bidder. In order to avoid such possibilities, in all real-world clock auctions of which we are aware, the auctioneer has reported only end-of-round aggregate demands, and not the individual demands of bidders.

\subsection{Reserve Pricing}

A reserve price is a second important instrument to address limited competition. It does this in two ways. First, it reduces the incentive for collusion by limiting the maximum gain from collusion. Bidders must pay at least the reserve price no matter how effective their collusion. Second, the reserve price guarantees that the seller receives a significant fraction of the goods' value, even when competition is weak. Reserve prices are easily implemented in clock auctions. Most commonly, the initial clock price serves as a reserve. Bidders are unable to express demands at prices below the reserve.

Alternatively, the auctioneer can start the auction at a low price but apply a secret reserve. A given product does not clear until the demand is less than or equal to the supply and the reserve price (which is not announced) is met. This approach was applied successfully in the September 2003 EDF auction.

More generally, the seller may wish to adjust supply in response to bids (McAdams 2002; Ausubel and Cramton 2004). In a clock auction, a supply adjustment is most easily accomplished by specifying an explicit upwardsloping supply curve. This has the effect of expanding the quantity offered for sale when there is ample competition, but reducing the quantity offered (and implicitly introducing a reserve-like mechanism) when there is insufficient competition. 


\subsection{Efficient Pricing}

The pricing rule is a final instrument to address market power. Most auctions for divisible goods use uniform pricing: all units of a given product sell at the same market-clearing price. The difficulty with this pricing rule is that it creates the incentive for bidders to engage in demand reduction-bidding a quantity less than true demand at every price (Ausubel and Cramton 2002). Moreover, the demand-reduction incentive increases in the quantity demanded: larger bidders shade more and smaller bidders shade less. This differential shading creates an inefficiency in which small bidders win too much and large bidders win too little.

This inefficiency can be completely avoided in a clock auction by making a simple change in the pricing rule, as proposed by Ausubel (1997, 2002). For homogeneous goods, each unit is awarded at the price at which it is "clinched," that is, at the point where it becomes mathematically impossible for the bidder not to win the unit. For example, suppose that there are four identical items and three bidders, each demanding two units. If one bidder reduces his quantity bid from two units to one, then each of the other two bidders "clinches" one unit at the current clock price. The clock continues to ascend in order to determine the allocation and price of the remaining two units. Under this modified rule, the clock auction yields the same pricing as in the (sealed-bid) Vickrey auction (Vickrey 1961). Bidders now have an incentive to bid their true demands and an efficient allocation is obtained.

Although the Ausubel auction eliminates the assignment inefficiency created by demand reduction, it does not solve the revenue problems stemming from market power. Under either uniform or Vickrey pricing, bidders pay less when there is less competition. Hence, the other tools-information policy and reserve pricing — need to be used in combination with Vickrey pricing to address the revenue problems created by limited competition.

\section{Conclusion}

A simultaneous clock auction is a powerful tool for auctioning many divisible goods. The auctioneer announces a price for each good, and bidders express the quantities of goods that they desire at the current prices. The prices of the goods are incremented in relation to their respective excess demands. The process repeats until there is no excess demand for any good. At that point bidders are awarded their quantities bid at the final prices.

While the underlying theory utilizes continuous clocks, bidding in realworld dynamic auctions normally occurs in discrete rounds. This, however, does not pose much of an obstacle to efficiency. What is needed is a mechanism for 
bidders in each round to express demands at intermediate prices between the starting and ending prices. This intraround bidding enables the efficiency gains of continuous clocks. All that is lost is the within-round price discovery, but this is much less important than the price discovery between rounds, which is retained - and even enhanced — by a pause in the bidding, allowing time for the bidders to update their strategies in light of the new information.

An important element of the auction design is the activity rule, which limits bidding behavior in a way that is consistent with reasonable preferences. This promotes price discovery. In clock auctions for a single good, all that is required is a monotonicity condition: as prices rise, bidders can maintain the same quantity or reduce quantity, but cannot increase quantity. Thus, the bidding must be consistent with a downward sloping demand curve. With many goods that are close substitutes, such a condition would be excessively harsh. In such auctions, it is best to group goods together that are strong substitutes and impose the monotonicity on the bidder's total quantity within the group. This enables buyers to arbitrage freely across goods within the group. Seller substitution also can be addressed. For example, the seller can specify price spreads across goods in a group and only specify the total quantity for the group. The quantity sold of each good within a group is then determined by both the buyers' and seller's preferences.

Market power is a final practical concern. This is addressed with three instruments available to the auctioneer. An information policy is established to limit the bidders' ability to adopt collusive strategies. Reserve prices and supply adjustments are used to limit the incentive for collusion and to guarantee significant revenue to the seller even if competition is weak or collusion is effective. Finally, the seller can switch from uniform pricing to Vickrey pricing to avoid the inefficiencies created by market power under uniform pricing. When combined properly these three tools can address both the efficiency and revenue losses arising from market power.

Although simultaneous clock auctions are new, they have already been applied in high-stakes auctions in half a dozen countries. Applications have included electricity auctions in France, the United States, and Belgium, gas auctions in Germany and Austria, and environmental auctions in the United Kingdom. Still the study and use of simultaneous clock auctions can be greatly expanded. The auction of Treasury and other financial securities is a compelling future application.

\section{References}

Ausubel, Lawrence M. (1997). “An Efficient Ascending-Bid Auction for Multiple Objects.” University of Maryland Working Paper 97-06 (forthcoming,American Economic Review). 
Ausubel, Lawrence M. (2002). “An Efficient Dynamic Auction for Heterogeneous Commodities." Working paper, University of Maryland.

Ausubel, Lawrence M. and Peter Cramton (2002). "Demand Reduction and Inefficiency in Multi-Unit Auctions." University of Maryland Working Paper 96-07.

Ausubel, Lawrence M. and Peter Cramton (2004). "Vickrey Auctions with Reserve Pricing." Economic Theory, forthcoming.

Ausubel, Lawrence M., Peter C. Cramton, and Wynne P. Jones (2002). "System and Method for an Auction of Multiple Types of Items." International Patent Application No. PCT/ US02/16937.

Compte, Olivier and Philippe Jehiel (2000). "On the Virtues of the Ascending Price Auction." Working paper, CERAS-ENPC.

Cramton, Peter and Jesse Schwartz (2002). "Collusive Bidding in the FCC Spectrum Auctions." Contributions to Economic Analysis \& Policy, 1:1. Available at 〈www. bepress.com/bejeap/contributions/vol1/iss1/art11).

Laan, Gerard van der and A. J. J. Talman (1987). "Adjustment Processes for Finding Economic Equilibria." In The Computation and Modelling of Economic Equilibria, edited by A. J. J. Talman and Gerard van der Laan. Amsterdam: North-Holland.

McAdams, David (2002). "Modifying the Uniform-Price Auction to Eliminate 'CollusiveSeeming Equilibria'.” Working paper, Massachusetts Institute of Technology.

Milgrom, Paul (2004). Putting Auction Theory to Work. Cambridge University Press.

Samuelson, Paul A. (1941). "The Stability of Equilibrium: Comparative Statics and Dynamics." Econometrica, 9, 97-120.

Varian, Hal R. (1981). "Dynamical Systems with Applications to Economics." In Handbook of Mathematical Economics, Vol. I, edited by Kenneth J. Arrow and Michael D. Intriligator. Amsterdam: North-Holland.

Vickrey, William (1961). "Counterspeculation, Auctions, and Competitive Sealed Tenders." Journal of Finance, 16, 8-37.

Walras, Léon (1874). Eléments d'Économie Politique Pure. Lausanne: Corbaz. 